\title{
Case Study: A patient comes to Emergency with slurring speech
}

\author{
EloísaUrrechaga* \\ *Hospital Galdakao - Usansolo, Division of medicinal laboratory, Galdakao,Vizcaya,Spain
}

Received: November 7, 2016; Accepted: February 21, 2017; Published: March 2, 2017

*Corresponding author: EloísaUrrechaga,Hospital Galdakao - Usansolo, Division of medicinal laboratory,Galdakao,Vizcaya,Spain, E-mail: ELOISAMARIA.URRECHAGAIGARTUA@osakidetza.eus

\begin{abstract}
A 72 years old male was brought to the emergency when his wife noticed that he suddenly was slurring his speech. The patient was diagnosed of transient ischemic attack (TIA), and started therapy with antiplatelet agents, combination aspirin/dipyridamole. Next day a complete analysis was requested, including fasting serum cholesterol, coagulation tests and HbA1c for type 2 diabetes mellitus screening. On evaluation of chromatogram a variant $\mathrm{Hb}$ was incidentally found, $16 \%$ in concentration, which was flagged as $\mathrm{HbC}$, because it eluted at the time of this common variant, in this system. Molecular analysis revealed an alpha variant, $\mathrm{Hb}$ Setif. $\mathrm{HbS}$ etif is characterized by an alpha 94 (G1) Asp > Tyr substitution. This mutation leads to a drastic change together in polarity and in steric hindrance and thus causes an unstable and functionally modified protein. This change is associated with some abnormal physic -chemical properties of the molecule especially an altered oxygen affinity; moreover the oxy-form has a strong tendency to polymerize, causing sickling of erythrocytes in vitro. Our patient presented analytical data which discarded risk factors of TIA, and the typical pattern of mild hemolysis was not considered at the beginning. The incidental finding of a variant is clinically relevant and must be reported, and the potential risks evaluated.
\end{abstract}

\section{Background}

Hemoglobinopathies are the most common inherited disorders in humans and are thus the target of screening programs worldwide. Over $1000 \mathrm{Hb}$ variants have been described, $\mathrm{Hb} \mathrm{S}$ is, worldwide, the most frequent, clinically severe $\mathrm{Hb}$ variant. The discovery of HbS by Linus Pauling and colleagues in 1949 was the first demonstration that the production of an abnormal protein could be the cause of a genetic disorder. So was born the notion of "molecular disease" and Sickle Cell Disease (SCD) was the first example. In 1956, Vernon Ingram identified the abnormality in the amino acid sequence of the $\beta$-globin chain ( $\beta 6 \mathrm{Glu} \rightarrow \mathrm{Val})$.

The initial observation of the abnormal morphology of red blood cells that defines the disorder. During the deoxygenation which follows the passage of RBCs in the microcirculation the $\mathrm{Hb}$ molecule undergoes a conformational change. In HbS, replacement of the hydrophilic glutamic acid at position 6 in the $\beta$ - globin chain by the hydrophobic valine residue makes that this last one establishes hydrophobic interactions with other hydrophobic residues on the $\beta$-globin chain of another deoxy $\mathrm{HbS}$ molecule. A polymer forms and lengthens in helical fibres which, grouped together, stiffen, and induce the characteristic SSRBC shape change, classically in the shape of a sickle.

A kind of pseudo sickling can be associated to other aminoacid change; whenthemodified residue is involved in a bond changing during the oxy - deoxy transition. This is the case for Hb Setif, an unusual incidental finding in a patient who suffered a stroke.

\section{Clinical and Laboratory findings}

A 72 years old male was brought to the emergency when his wife noticed that he suddenly was slurring his speech with his face drooping on one side.

The patient had a history of prostatism, he never smoked, and the consumption of alcohol was less than $50 \mathrm{cc}$ of wine /day.

In the emergency department, the patient is alert and oriented. Normal vital and electrocardiogram was normal.

He is still complaining of "numbness" of the right side of face and down the right arm. His speech is clear, can follow commands, not have a headache and denies any nausea or vomiting.

A head Computed Tomography (CT) scan showed no acute intracranial change and Magnetic Resonance Imagery (MRI) is within normal limits.

The patient was admitted for a neurology evaluation, Magnetic Resonance Angiography (MRA) of the brain,. Upon admission to the unit, the symptoms have resolved.

The patient was diagnosed of Transient Ischemic Attack (TIA), and started therapy with antiplatelet agents, combination aspirin/dipyridamole.

Next day a complete analysis was requested, including fasting serum cholesterol, coagulation tests and HbA1c for type 2 diabetes mellitus screening.

\section{Analytical data}

Glucose $90 \mathrm{mg} / \mathrm{dL}$ (reference 76-110), Urea $36 \mathrm{mg} / \mathrm{dL}$ (reference 10-50), 
creatinin $1.04 \mathrm{mg} / \mathrm{dL}$ (reference 0.6-1.3), total Protein $6.8 \mathrm{gd} / \mathrm{L}$, (reference 6.3-8.3)

Sodium $141 \mathrm{mEql} / \mathrm{L}$ (reference 135-145), Potasium 4.4 $\mathrm{mEql} / \mathrm{L}$ (reference 3.5-4.5)

Calcium 9.2 mg/dL (reference 9.0-10.5), Cholesterol185 mg/ dL (reference 150-200)

Bilirrubin $1.3 \mathrm{mg} / \mathrm{dL}$, (reference 0.2-1.8), AST $27 \mathrm{IU} / \mathrm{L}$ (reference 5-37), ALT $30 \mathrm{U} / \mathrm{L}$ (reference 5-40), LHD $260 \mathrm{U} / \mathrm{L}$ (reference 110-210), Hb A1c $6.1 \%$ (normal <6.5\%)

\section{ESR $9 \mathrm{~mm} / \mathrm{h}$ (reference 1-20)}

WBC $5.1 \times 10^{9} / \mathrm{L}, \mathrm{RBC} 4.11 \times 10^{12} / \mathrm{L}$, Hemoglobin $120 \mathrm{~g} / \mathrm{L}$, Hematocrit 0.36 L/L, MCV 84.8 fL, MCH 29,6 pg, MCHC 330 g/L, RDW $13.6 \%$, Platelets $255 \times 10^{9} / \mathrm{L}$, MPV 10.5 fL.Reticulocyte count $3.5 \%, 144 \times 10^{9} / \mathrm{L}$. Automated WBC differential count: normal.

Prothrombin Time (PT) 12.9 seconds, international normalized ratio (INR) 1.10; Activated Partial Thromboplastin Time (aPTT) 33 sg.

HbA1c was analyzed with HPLC (ARKRAY ADAMS ${ }^{\mathrm{TM}}$ A1c HA 8180 Variant mode). On evaluation of chromatogram a variant $\mathrm{Hb}$ was incidentally found, $16 \%$ in concentration, which was flagged as $\mathrm{HbC}$, because it eluted at the time of this common variant, in this system.

Nevertheless the \% suggested a mutation on alpha genes, and the sample was sent to a reference laboratory for the identification of the $\mathrm{Hb}$.

The result was HbSetif [94 (G1) Asp $\rightarrow$ Tyr].

\section{Discussion \& Key points}

Except sex and age the patient had no risk factors for ischemia:

Age. Sex.Family history.

Cigarette smoking. Heavy drinking. Use of illicit drug

Prior transient ischemic attack.

Sickle cell disease.

High blood pressure. High cholesterol.

Cardiovascular disease. Peripheral Artery Disease (PAD).

Diabetes.

The normal coagulation tests discarded Antiphospholipid Syndrome; high cholesterol and diabetes were discarded as well.

Incidental finding of Variant Hbs while diabetes evaluation is frequent in our daily practice. Due to immigration heterozygous $\mathrm{HbS}$ is the most frequent hemoglobinopathy in our area.

$\mathrm{Hb}$ Setifis an alpha chain Variant $\mathrm{Hb}$. Hb Setif is much less soluble than $\mathrm{Hb} \mathrm{A}$ and induces pseudosickling of the red cells in vitro. This hemoglobin presents in a low percent (12-17\%). $\mathrm{Hb}$ Setif has been found in Algeria, Iran, Lebanon, Saudi Arabia, Turkey, Italy, Malta, and Cyprus; only another case was reported in Spain.
This mutant is an $\alpha$ chain mutant, and it is known that this category has a much lower frequency than $\beta$ chain mutants; the intra-erythrocytic disorders are minor when compared to $\beta$ chain mutant ones.

HbSetif is characterized by an alpha 94 (G1) Asp > Tyr substitution [1].

This mutation leads to a drastic change together in polarity and in steric hindrance and thus causes an unstable and functionally modified protein.

The Asp -> Tyr substitution induces a conformation change of the sequence around it,

From a random coil in $\mathrm{HbA}$ to a $\beta$ sheet in $\mathrm{Hb}$ Setif.

Since $\beta$-sheet structures are well known to give rather stable hydrophobic interactions, this could result inabnormal binding between sub-units or molecules of $\mathrm{Hb}$. Such a $\beta$-sheet- $\beta$-sheet interaction in HbSetifwould cause aggregation between two a-chains, leading to an $\alpha$ l $\alpha$ l contact in HbSetif instead of the $\alpha 1 /$ $\beta 2$ contact in $\mathrm{HbA}$.

This change is associated with some abnormal physicochemical properties of the molecule especially an altered oxygen affinity; moreover the oxy-form has a strong tendency to polymerize, causing sickling of erythrocytes in vitro.

The modified residue of $\mathrm{Hb}$ Setif is involved in a bond changing during the oxy-deoxy transition confers a lowered oxygen affinity of $\mathrm{Hb}$ Setif.

Pseudosicking consists of bundles of twisted fibers. Those fibers and early stages of molecular aggregation increase rigidity and might expect to find infarctions in hypertonic environment similar to those seen in sickle cell disease or trait [2].

The sickle red blood cell can contribute to the pathogenesis of stroke via abnormal adherence to the vascular endothelium and by hemolysis, which results in endothelial cell activation hypercoagulability.

Sickled red blood cells are abnormally adherent to the vascular endothelium. Adhesion of deformed red cells in the microcirculation leads to the trapping of denser, less deformable cells,79 which prolongs transit time and enables polymerisation of sickle haemoglobin80 resulting in vasoocclusion. Since erythrocyte-endothelial inter actions seem to be most prominent in the microvasculature, this mechanism could be prominent for cerebral infarction [3].

HbSetif can cause a pseudosickling in vitro, has not been proven in vivo; but the higher stiffness can induce abnormal interactions with the vascular endotheliumabnormal adherence to the vascular endothelium and hemolysis result in a proinflammatory state manifested, in part, by leucocyte adhesion and platelet aggregation.

Sickle cell trait human erythrocytes are significantly stiffer than normal; chronic hemolysis, and therefore the effect of free $\mathrm{Hb}$ may be paramount in stroke pathogenesis [4]. 
A new mechanism, involving free $\mathrm{Hb}$ interaction with Von Willebrand Factor (VWF), recently proposed can explain the molecular basis of the hypercoagulability when hemolysis is present. Free hemoglobin increases von Willebrand factormediated platelet adhesion: $\mathrm{Hb}$ interacts directly with the A1 domain. And affects the GPIb $\alpha$-VWF interaction. Extracellular $\mathrm{Hb}$ directly affects the GPIb $\alpha$-VWF interaction in thrombosis, and describes another mechanism by which hemolysis is connected to thrombotic events [5].

Our patient presented analytical data which discarded risk factors of TIA, and the typical pattern of mild hemolysis was not considered at the beginning.

The incidental finding of a variant is clinically relevant and

\section{References}

1. Wajcman H, Belkhodja O, Labie D. Hb Setif: Gl (94) $\alpha$ Asp > Tyr a new $\alpha$ chain hemoglobin variant with substitution of the residue involved in a hydrogen bond between unlike subunits. FEBS Lett. 1972;27(2):298300.

2. Charache S, Raik E, Holtzclaw D, Hathaway PJ, Powell E, Fleming P. Pseudosickling of Hemoglobin Setif. Blood. 1987;70(1):237-242.

3. Switzer JA, Hess DC, Nichols FT, Adams RJ. Pathophysiology and treatment of stroke in sickle-cell disease: present and future. Lancet Neurol. 2006;5(6):501-512.

4. Bess CD, Krause MA. Abnormal PfEMP1/knob display on Plasmodium falciparum-infected erythrocytes containing hemoglobin variants: fresh insights into malaria pathogenesis and protection. Microbes Infect. 2012;14(10):851-862. doi: 10.1016/j.micinf.2012.05.006

5. Da Q, Teruya M, Guchhait P, Teruya J, Olson JS, Cruz MA. Free hemoglobin increases von Willebrand factor-mediated platelet adhesion in vitro: implications for circulatory devices. Blood. 2015;126(20):2338-2341. doi: 10.1182/blood-2015-05-648030

6. Urrechaga E. Incidental Detection of $\mathrm{Hb}$ Disorders During HbA1c Analysis. J Diabetes Sci Technol. 2015;9(3):713. doi: $10.1177 / 1932296815574727$ 